\title{
Estimativa da evapotranspiração de referência em Campina Grande, Paraíba ${ }^{1}$
}

\author{
Francisco de A. N. Henrique ${ }^{2}$ \& Renilson T. Dantas ${ }^{2}$
}

\begin{abstract}
RESUMO
Deste trabalho se objetivou estimar, através de uma fórmula empírica, a evapotranspiração de referência diária no município de Campina Grande, PB (EToT), utilizando-se a amplitude térmica diária. Estimou-se, também, a ETo diária usando-se os métodos de FAO-Penman-Monteith (EToPM), Hargreaves (EToH) e Linacre (EToL), sendo depois correlacionados com a EToT proposta na pesquisa, por estação do ano; para isto foram coletados valores diários das temperaturas, umidade relativa do ar, radiação solar, precipitação, pressão atmosférica e velocidade do vento a $2 \mathrm{~m}$ da Estação Meteorológica Automática, instalada na Universidade Federal de Campina Grande (UFCG), no período de 1 de janeiro a 31 de dezembro de 2004. Tais valores foram registrados no intervalo de $10 \mathrm{~min}$. Verificou-se que as correlações da EToT com EToH, obtiveram o melhor ajuste, cujos coeficientes foram: $R^{2}=0,7882$ (no ano), $R^{2}=0,9796$ (no verão), $R^{2}=0,8253$ (no outono), $R^{2}=0,878$ (no inverno) e $R^{2}=0,914$ (na primavera). Conclui-se que os métodos de EToPM e EToL não se ajustaram bem para Campina Grande, PB, o que evidencia que a EToT poderá ser utilizada na forma proposta para estimativa da ETo desta localidade. Das equações propostas, a única restrição é o EToT para o verão caso em que se recomenda o uso da equação anual.
\end{abstract}

Palavras-chave: precipitação, demanda climática, amplitude térmica

\section{Estimate of reference evapotranspiration in the city of Campina Grande, Paraíba state, Brazil}

\begin{abstract}
The objective of this study was to estimate, using an empiric formula, the daily reference evapotranspiration in the city of Campina Grande, Paraiba State, Brazil (EToT), using the daily thermal amplitude. The ETo was also estimated using the methods of FAO-Penman-Monteith (EToPM), Hargreaves (EToH) and Linacre (EToL), being correlated later with EToT proposed in this research by season. For that, daily values of temperature, air relative humidity, solar radiation, precipitation, atmospheric pressure and wind speed at $2 \mathrm{~m}$, were taken using the Automatic Meteorological Station installed in the Universidade Federal de Campina Grande (UFCG), over the period of January 1 to December 31, 2004. These values were registered at intervals of $10 \mathrm{~min}$. It was verified that the correlations of EToT with EToH had better adjustments where the coefficients were: $R^{2}=0.7882$ (for the year), $R^{2}=0.9796$ (for the summer), $R^{2}=0.8253$ (for the autumn), $R^{2}=0.878$ (for the winter) and $\mathrm{R}^{2}=0.914$ (for the spring). The methods of EToPM and EToL did not adjust well for Campina Grande, which indicates that EToT can be used there in the form proposed for estimate of ETo. The only restriction among the proposed equations is EToT for summer, in which case the use of the annual equation is recommended.
\end{abstract}

Key words: precipitation, climatic demand, thermal amplitude

1 Parte da Dissertação de Mestrado do primeiro autor, financiada pelo CNPq

2 UACA/UFCG, Av. Aprígio Veloso, 882, CEP: 58109-970, Campina Grande, PB. Fone: (83) 3310-1054. E-mail: nunesdeassis@pop.com.br, Fone: (83) $3310-1202$. E-mail: renilson@dca.ufcg.edu.br. 


\section{INTRODUÇÃO}

Na agricultura, informações quantitativas da evapotranspiração são de grande importância na avaliação da severidade, distribuição e freqüência dos déficits hídricos, elaboração de projetos e manejo de sistemas de irrigação e drenagem.

Para o agreste paraibano, onde está localizada a cidade de Campina Grande, PB (ou regiões circunvizinhas), a quantificação da evapotranspiração assume particular significação em virtude dos déficits hídricos ao longo do ano, constituindo séria limitação à produção agrícola e permanente fonte de risco agrícola, em quase toda a região, principalmente em áreas secas cujas características climáticas se aproximam da semi-aridez.

Segundo Penman (1963), as limitações dos métodos empíricos são reconhecidas tanto por seus críticos como por seus autores mas, enquanto prosseguem as buscas por melhores soluções, eles podem fornecer valores de consumo de água para uso em balanço hídrico e, nas melhores condições, valores que são, pelo menos, tão precisos quanto os que podem ser obtidos por medidas diretas no campo e o fazem de modo mais fácil. Pode-se acrescentar que possuir informações de demanda de água através de um método empírico, mesmo o mais simples, é melhor do que não possuir nenhuma informação. Sabe-se, ainda, que fórmulas empíricas mais simples são as mais usadas, não por sua universalidade ou precisão mas devido à não existência de medidas de superfície que permitem o uso de métodos mais consistentes.

Durante uma década a FAO (Food and Agriculture Organization) adotou o conceito e a forma de cálculo da evapotranspiração de referência proposta por Doorenbos \& Pruitt (1977), Allen et al. (1989), comparando a evapotranspiração de referência obtida pelos métodos de Penman, Kimberly-Penman, Penman corrigido e Penman-Monteith; com medições lisimétricas, observaram que o modelo de PenmanMonteith foi o que melhor se ajustou às medições diárias e mensais e concluíram que a alfafa se ajusta melhor que a grama como cultura padrão na determinação da evapotranspiração de referência, visto que sua altura e rugosidade se aproximam da maioria das culturas agrícolas. A calibração da equação de Penman provocou vários problemas na definição da cultura de referência. Alguns pesquisadores argumentaram que as variedades de grama e suas características morfológicas não são padronizadas para diferentes condições climáticas e a alfafa apresenta problemas de variedade e manejo (Allen et al., 1994).

Adotou-se como padrão, em 1998, a estimativa da evapotranspiração de referência à FAO/56. Silva et al. (2001) realizaram a estimativa da evapotranspiração de referência pelos métodos de Penman-Monteith - FAO/56, Hargreaves e Tanque "Classe A", com dados da estação climatológica de Campina Grande, PB; foram utilizados os dados das temperaturas máxima e mínima do ar, insolação e velocidade do vento e desse estudo, eles deduziram que a evapotranspiração obtida pelo método de Penman-Monteith superestima os valores obtidos pelo método de Hargreaves em períodos mensais e semanais. Na ausência de variáveis climatológicas necessárias para a aplicação do modelo da FAO, a equação de Hargreaves pode ser utilizada com precisão na estimativa da evapotranspiração de referência; enfim, os autores concluíram que a estimativa da evapotranspiração com base no Tanque "Classe A" deve ser realizada com cautela.

Silva et al. (2005) também avaliaram a eficiência dos métodos de estimativa de referência (Penman-Monteith (FAO/56), Hargreaves, Jensen-Haise, Linacre, Makkink, Priestley e Taylor, Tanque "Classe A" e Thornthwaite utilizando o sistema de estimativa da evapotranspiração SEVAP além de dados médios mensais do ano de 2002 da estação climatológica de Petrolina, PE; usando o coeficiente de determinação e a média e desvio padrão, concluíram que na ausência de dados de insolação e velocidade do vento a evapotranspiração de referência pelo método de Penman-Monteith pode ser substituída, com razoável precisão, pelo método de Hargreaves. Os métodos de Linacre e de Thornthwaite não apresentaram resultados satisfatórios na estimativa da ETo, em escala diária.

Oliveira \& Volpe (2003) compararam estimativas de evapotranspiração de referência (ETo) na região de Jaboticabal, SP, pelos métodos de Penman e Penman-Monteith (FAO), em períodos seco e úmido, utilizando dados de estações meteorológicas convencional e automática. A análise dos resultados foi realizada no pacote estatístico SAS (1990), para o período diário (julho/1997 a junho/2002), através do modelo linear $\mathrm{y}=\mathrm{a}+\mathrm{bx}$, quando então verificaram que não houve tendência de superioridade nas estimativas da ETo para os métodos Penman e Penman-Monteith, independentes do período analisado e da fonte de dados utilizados.

Foi objetivo primordial, neste trabalho, estimar a ETo em Campina Grande, PB, a partir da amplitude térmica diária.

\section{MATERIAL E MÉTODOS}

Os valores diários das temperaturas, umidade relativa do ar, irradiação solar, precipitação, pressão e velocidade do vento a $2 \mathrm{~m}$, foram coletados da Estação Meteorológica Automática acoplada ao Datalogger modelo CR23X, da Unidade Acadêmica de Ciências Atmosféricas, instalada na Universidade Federal de Campina Grande (UFCG), localizada no município de Campina Grande, PB ( $7^{\circ} 14^{\prime} \mathrm{S}$, $35^{\circ} 54^{\prime}$ W e $551 \mathrm{~m}$ ) no período de 1 de janeiro a 31 de dezembro de 2004; tais valores foram registrados no intervalo de 10 minutos.

Com a planilha do Excel digitaram-se esses valores e se estimou, por estação do ano, a Evapotranspiração de Referência (ETo) pelos métodos empíricos citados a seguir.

\section{Método de Penman-Monteith (EToPM)}

$$
\mathrm{ET}_{0}=\frac{0,408 \Delta\left(\mathrm{R}_{\mathrm{n}}-\mathrm{G}\right)+\gamma\left(\frac{900 \mathrm{U}_{2}}{\mathrm{~T}+273}\right)\left(\mathrm{e}_{\mathrm{s}}-\mathrm{e}_{0}\right)}{\Delta+\gamma\left(1+0,34 \mathrm{U}_{2}\right)}
$$


O procedimento de cálculo de todas as variáveis da Eq. 1, está de acordo com o Boletim 56/FAO (Allen et al., 1998), donde: $\mathrm{D}$ é a declinação de curvatura $\left(\mathrm{K} \mathrm{Pa}^{\circ} \mathrm{C}^{-1}\right)$; $\mathrm{R}_{\mathrm{n}}$ é o saldo de radiação ( $\mathrm{MJ} \mathrm{m}^{-2}$ dia $\left.^{-1}\right)$; $\mathrm{G}$ é a densidade do fluxo de calor $\left(\mathrm{MJ} \mathrm{m}^{-2} \mathrm{dia}^{-1}\right)$; T é a temperatura $\left({ }^{\circ} \mathrm{C}\right)$; $\mathrm{U}_{2}$ é a velocidade do vento a $2 \mathrm{~m}\left(\mathrm{~m} \mathrm{~s}^{-1}\right)$; $\mathrm{e}_{\mathrm{s}}$ é a pressão de saturação do vapor (KPa); $e_{0}$ é a pressão real do vapor (KPa) e $\gamma$ é o fator psicométrico $\left(\mathrm{MJ} \mathrm{kg}^{-1}\right)$.

\section{Método de Hargreaves (EToH)}

Na ausência dos dados de radiação solar, umidade relativa e velocidade do vento, a evapotranspiração pode, em mm dia-1, ser estimada através da seguinte equação (Hargreaves, 1974):

$$
\mathrm{ET}_{0}=0,0023\left(\mathrm{~T}_{\text {med }}+17,8\right)\left(\mathrm{T}_{\mathrm{x}}-\mathrm{T}_{\mathrm{i}}\right)^{0,5} \mathrm{R}_{\mathrm{a}}
$$

em que: $T_{\text {med }}, T_{x}$ e $T_{i}$, em ${ }^{\circ} \mathrm{C}$, representam, respectivamente, as temperaturas média, máxima e mínima e $\mathrm{R}_{\mathrm{a}}$ é a radiação solar no topo da atmosfera (mm dia-1).

\section{Método de Linacre (EToL)}

A evapotranspiração de referência pelo método de Linacre, em mm dia-1 , pode ser obtida em função da altitude, latitude e das temperaturas diárias máxima, mínima e do ponto de orvalho, através da equação (Linacre, 1977):

$$
\mathrm{ET}_{0}=\frac{700 \frac{\mathrm{T}_{\mathrm{m}}}{(100-\varphi)}+15\left(\mathrm{~T}_{\mathrm{a}}-\mathrm{T}_{\mathrm{d}}\right)}{\left(80-\mathrm{T}_{\mathrm{a}}\right)}
$$

em que:

$$
\begin{aligned}
\mathrm{T}_{\mathrm{m}} & =\mathrm{T}_{\mathrm{a}}+0,006 \mathrm{z} \\
\mathrm{z} & - \text { altitude, } \mathrm{m} \\
\mathrm{T}_{\mathrm{a}} & - \text { temperatura média do ar, }{ }^{\circ} \mathrm{C} \\
\varphi & - \text { é a latitude local (graus), }{ }^{\circ} \\
\mathrm{T}_{\mathrm{d}} & - \text { é a temperatura de ponto de orvalho, }{ }^{\circ} \mathrm{C}
\end{aligned}
$$

\section{Método proposto pelo trabalho (EToT)}

Considerando-se o conhecimento adquirido através da literatura, constata-se haver uma relação bem próxima entre a evapotranspiração de referência e a amplitude térmica diária; neste sentido, procurou-se identificar esta relação de modo que, em se utilizando os métodos de estimativa descritos anteriormente e os dados de temperaturas máxima e mínima diários medidos em Campina Grande, PB, foram encontradas as seguintes equações:

$$
\begin{array}{ll}
\text { EToT }=0,885 \cdot\left(\mathrm{T}_{\mathrm{x}}-\mathrm{T}_{\mathrm{i}}\right)^{0,696} & \text { Equação anual } \\
\mathrm{EToT}=3,547 \cdot\left(\mathrm{T}_{\mathrm{x}}-\mathrm{T}_{\mathrm{i}}\right)^{0,072} & \text { Equação para o verão } \\
\mathrm{EToT}=0,881 \cdot\left(\mathrm{T}_{\mathrm{x}}-\mathrm{T}_{\mathrm{i}}\right)^{0,68} & \text { Equação para o outono } \\
\mathrm{EToT}=0,9 \cdot\left(\mathrm{T}_{\mathrm{x}}-\mathrm{T}_{\mathrm{i}}\right)^{0,907} & \text { Equação para o inverno } \\
\mathrm{EToT}=1,118 \cdot\left(\mathrm{T}_{\mathrm{x}}-\mathrm{T}_{\mathrm{i}}\right)^{0,609} & \text { Equação para a primavera }
\end{array}
$$

\section{Erro padrão da estimativa $\left(\mathrm{S}_{\mathrm{y}-\mathrm{x}}\right)$}

A expressão a seguir retorna o erro padrão do valor $\mathrm{y}$ previsto para cada $\mathrm{x}$ da regressão. De acordo com Spiegel (1977), a medida de dispersão em relação à reta de regressão de y para x é dada por:

$$
S_{y-x}=\sqrt{\frac{\sum\left(y-y_{\text {est }}\right)^{2}}{N}}
$$

donde:

$$
\begin{aligned}
\text { Yest }_{\text {- }} & \text { valores de EToT (y) correspondente a valores } \\
& \text { das três primeiras estimativas de evapotranspi- } \\
& \text { ração, citado anteriormente }(\mathrm{x}) \\
\mathrm{N} \text { - número de dias de cada estação } & \text { númo }
\end{aligned}
$$

\section{RESULTADOS E DISCUSSÃO}

\section{Evapotranspiração de referência no verão}

Tem-se na Figura 1, a comparação dos valores diários da evapotranspiração de referência (ETo) calculada pelos métodos de Penman-Monteith, Hargreaves, Linacre e EToT, no verão de 2004, em Campina Grande, PB; nela se observa a linha da EToT até aproximadamente o vigésimo dia do verão (começo de janeiro) ajustando-se melhor com a ETo de Hargreaves com $\mathrm{R}^{2}=0,9796$ (Figura 2); a partir de aproximadamente o vigésimo quinto dia, a EToT se ajustou melhor com as ETo de Hargreaves e Penman-Monteith; então, em todos os dias o ajuste menos adequado foi a ETo de Linacre. Verifica-se, ainda na Figura 1, que a estimativa da evapotranspiração de referência através do método de Hargreaves foi praticamente superior a 3,5 mm dia-1 em todo o verão, enquanto o método de Linacre estimou valores oscilando entre $2,0 \mathrm{~mm} \mathrm{dia}^{-1}$ e $2,5 \mathrm{~mm} \mathrm{dia}^{-1}$, o que demonstra diferenças significativas entre esses métodos; no verão, a EToT se ajustou bem aos métodos de Hargreaves e Penman-Monteith, com maior aproximação do método de Hargreaves.

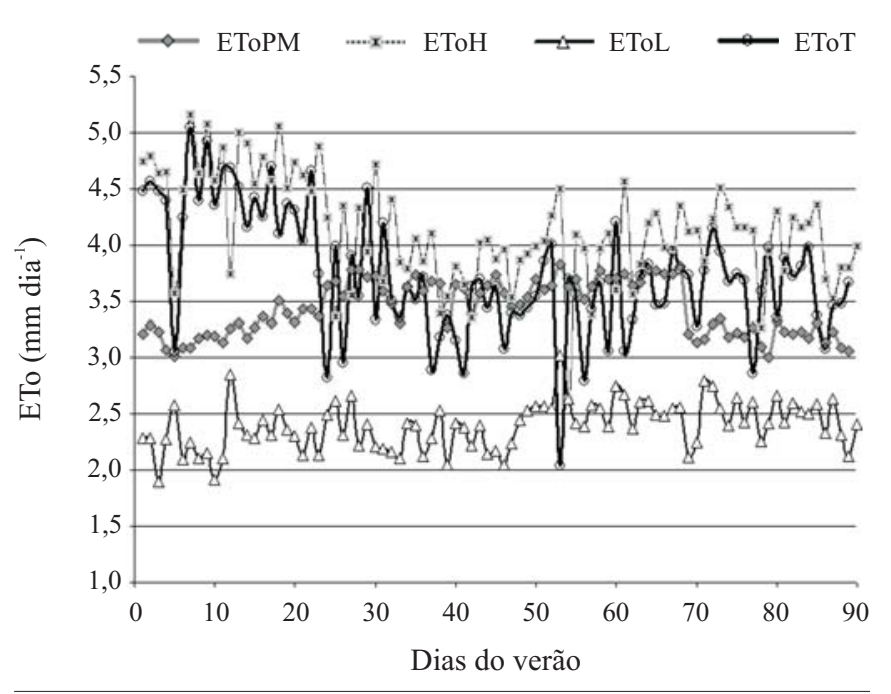

Figura 1. Evapotranspiração de referência calculada pelos métodos de Penman-Monteith, Hargreaves, Linacre e EToT no verão de 2004, em Campina Grande, PB 


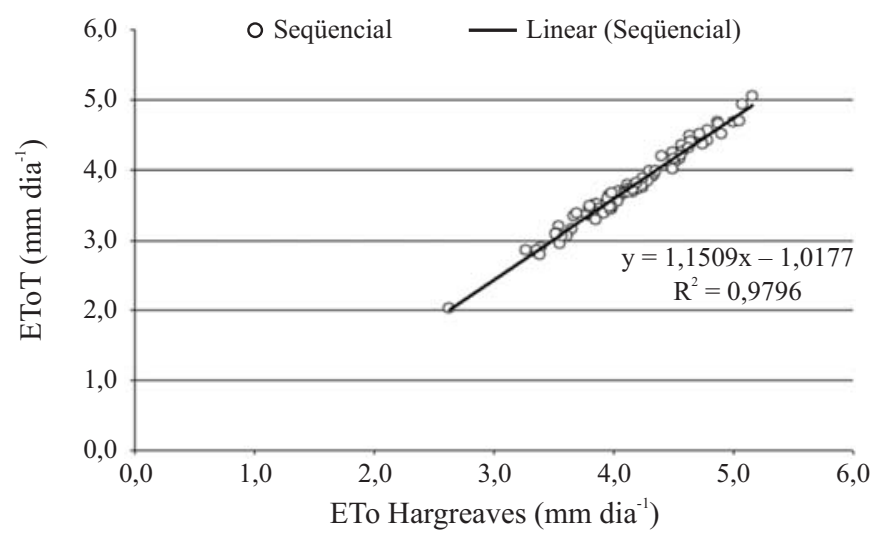

Figura 2. Regressão linear da EToT em função da ETo Hargreaves, no verão de 2004, em Campina Grande, PB

\section{Evapotranspiração de referência no outono}

Na Figura 3 se encontram os valores diários da evapotranspiração de referência calculada pelos métodos de Penman-Monteith, Hargreaves, Linacre e EToT, no outono de 2004, em Campina Grande, PB. Verifica-se que, em todos os dias desta estação, a EToT se correlacionou melhor com a estimativa de Hargreaves; raros os dias em que as linhas das ETo Penman-Monteith e Linacre se ajustaram melhor às ETo de Hargreaves e EToT, pois a EToH em função de EToT, obteve $\mathrm{R}^{2}=0,8253$ (Figura 4).

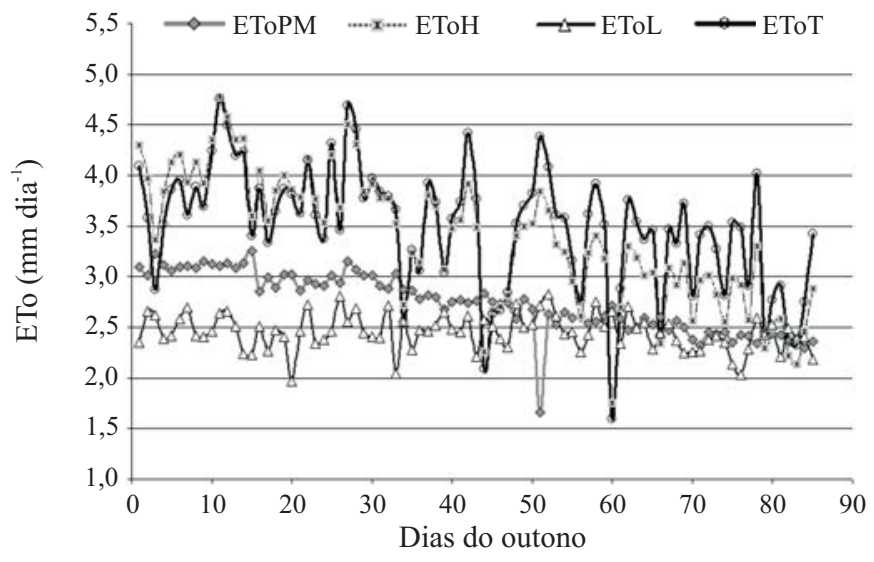

Figura 3. Evapotranspiração de referência calculada pelos métodos de Penman-Monteith, Hargreaves, Linacre e EToT no outono de 2004, em Campina Grande, PB

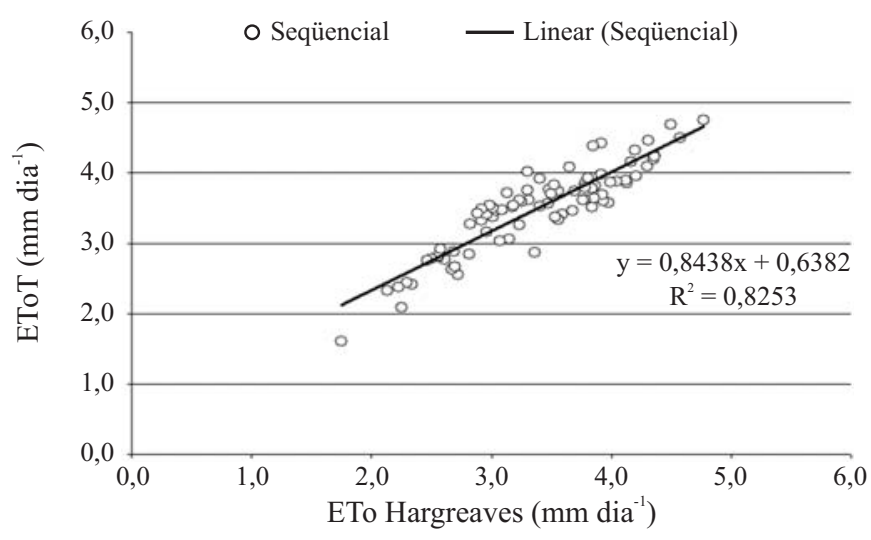

Figura 4. Regressão linear da EToT em função da ETo Hargreaves no outono de 2004, em Campina Grande, PB
Nesta estação se nota, ainda, que as estimativas EToT e EToH estão bem relacionadas enquanto outra relação também pode ser bem visualizada entre as estimativas de Penman-Monteith e Linacre, após os primeiros 30 dias do outono, principalmente no final da mesma.

\section{Evapotranspiração de referência no inverno}

Na Figura 5 se encontram os valores diários da evapotranspiração de referência calculada pelos métodos de Penman-Monteith, Hargreaves, Linacre e EToT, no inverno de 2004, em Campina Grande, PB. Nota-se que, em todos os dias da estação, a ETo se ajusta melhor com Hargreaves e também são raros os dias em que as linhas da ETo PenmanMonteith e ETo Linacre se ajustam melhor com EToT e ETo Hargreaves, pois este último obteve $\mathrm{R}^{2}=0,878$ (Figura 6). Verifica-se também que aproximadamente nos últimos 35 dias do inverno, as curvas da ETo Penman-Monteith e ETo Linacre apresentaram as maiores discrepâncias; no inverno deste ano em Campina Grande, os valores da EToT se ajustaram muito bem aos valores da EToH; ainda se constata que as estimativas EToPM e EToL mantiveram boa relação até aproximadamente o final de julho; outro aspecto significativo se liga ao fato de que, em ambos os casos, elas subestimaram a EToT, praticamente em todo o período.

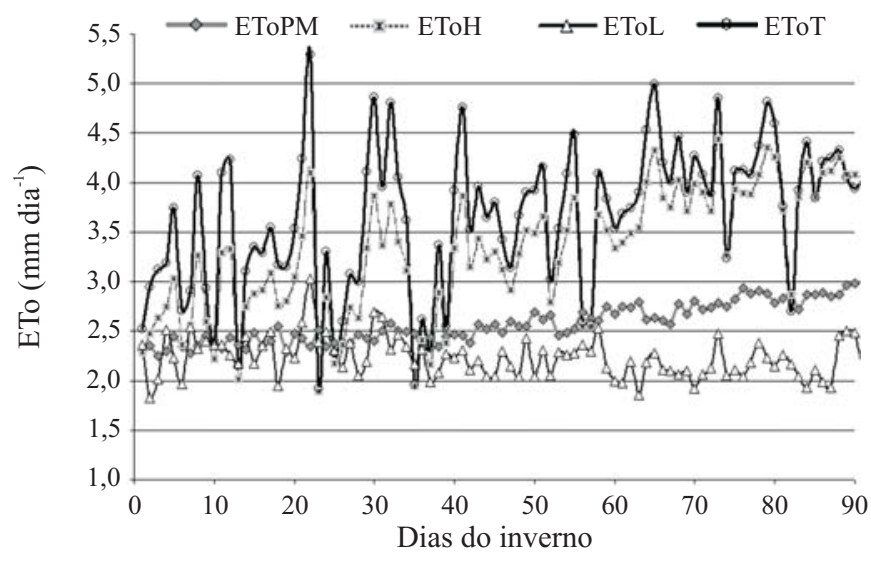

Figura 5. Evapotranspiração de referência calculada pelos métodos de Penman-Monteith, Hargreaves, Linacre e EToT no inverno de 2004, em Campina Grande, PB

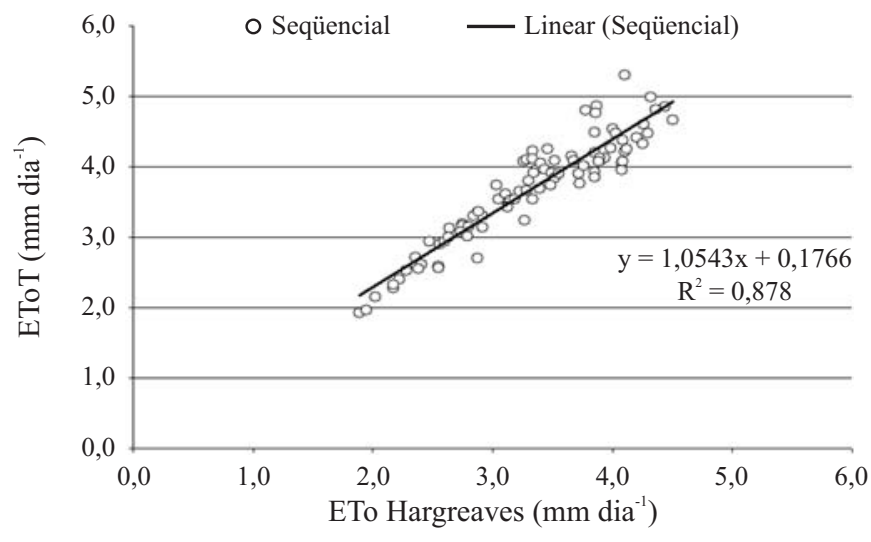

Figura 6. Regressão linear da EToT em função da ETo Hargreaves no inverno de 2004, em Campina Grande, PB 


\section{Evapotranspiração de referência na primavera}

A Figura 7 mostra os valores diários da evapotranspiração de referência calculada pelos métodos de PenmanMonteith, Hargreaves, Linacre e EToT na primavera de 2004, em Campina Grande, PB; aqui se observa claramente a melhor correlação entre a EToT, em função da ETo Hargreaves (que variaram aproximadamente entre $3,3 \mathrm{~mm} \mathrm{dia}^{-1}$ a $5,4 \mathrm{~mm} \mathrm{dia}^{-1}$ ) com $\mathrm{R}^{2}=0,914$ (Figura 8), enquanto durante todos os dias desta estação as linhas da ETo Penman-Moteith (que variou entre $2,8 \mathrm{~mm} \mathrm{dia}^{-1}$ a 3,8 $\mathrm{mm} \mathrm{dia}^{-1}$ ) e ETo Linacre $\left(1,5 \mathrm{~mm} \mathrm{dia}^{-1}\right.$ a 2,5 $\left.\mathrm{mm} \mathrm{dia}^{-1}\right)$ compuseram os métodos de estimativas mais distantes da EToT.

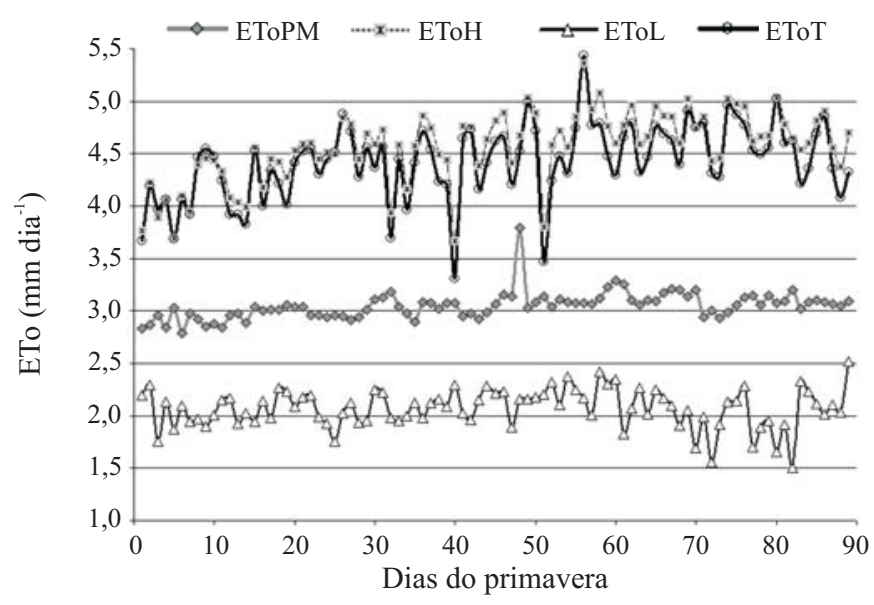

Figura 7. Evapotranspiração de referência calculada pelos métodos de Penman-Monteith, Hargreaves, Linacre e EToT na primavera de 2004, em Campina Grande, PB

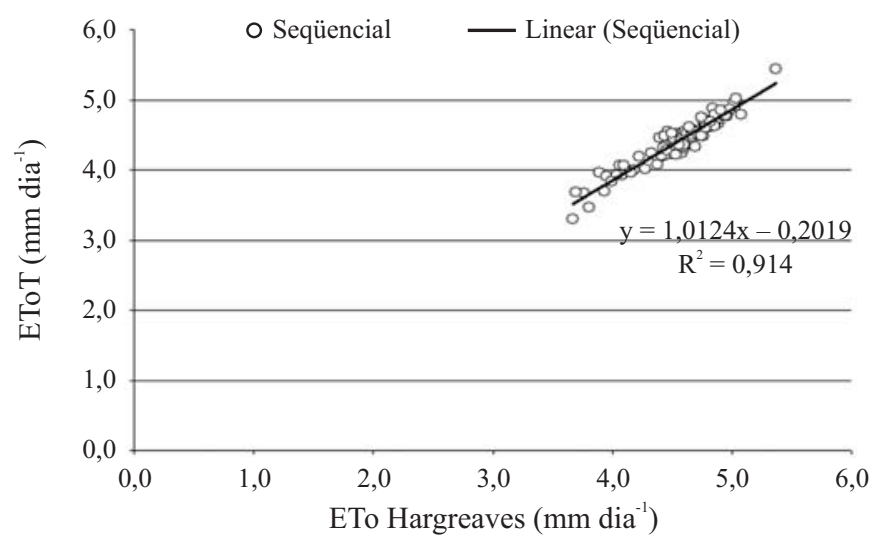

Figura 8. Regressão linear da EToT em função da ETo Hargreaves na primavera de 2004, em Campina Grande, PB

\section{Correlação dos métodos estimados}

Na Tabela 1 se encontram a correlação entre os métodos de estimativa da evapotranspiração de referência utilizados neste trabalho, os coeficientes a e b da regressão linear e o coeficiente de determinação $\mathrm{R}^{2}$, para cada caso. Verifica-se, através da tabela, haver correlação diretamente proporcional entre EToH x EToPM, EToH x EToT, EToPM x EToL e EToPM x EToT, embora os valores de evapotranspiração de referência, estimados pela EToPM, sejam sempre inferiores àqueles estimados pela $\mathrm{EToH}$, enquanto isto ocorre em relação a EToL para EToL $\leq 3,0 \mathrm{~mm}$.

Por intermédio da mesma tabela se verifica, também, haver correlação inversa proporcional entre EToH x EToL e EToL x EToT; por outro lado se constata, na tabela mencionada, que a única correlação aceita do ponto de vista estatístico, é EToH x EToT; em outras palavras, na impossibilidade da utilização da EToT na estimativa da evapotranspiração de referência em Campina Grande, a mesma poderá ser estimada pelo método de Hargreaves, com grau de confiança aceitável neste contexto.

Tabela 1. Correlação e coeficientes de estimativa de evapotranspiração de referência estimada pelos métodos de Hargreaves (EToH), PenmanMonteith (EToPM), Linacre (EToL) e o proposto pelo trabalho (EToT), durante 0 ano de 2004, em Campina Grande, PB

\begin{tabular}{|c|c|c|c|c|}
\hline & & \multicolumn{3}{|c|}{ Coeficiente } \\
\hline \multicolumn{2}{|c|}{ Correlação } & Angular & Linear & Determinação \\
\hline$y$ & $x$ & a & b & $R^{2}$ \\
\hline $\mathrm{EToH}$ & EToPM & 1,1203 & 0,5462 & 0,3423 \\
\hline $\mathrm{EToH}$ & EToL & $-0,6767$ & 5,3964 & 0,0485 \\
\hline $\mathrm{EToH}$ & EToT & 0,991 & 0,0496 & 0,77882 \\
\hline EToPM & EToL & 0,0558 & 2,8233 & 0,0012 \\
\hline EToPM & EToT & 0,1267 & 2,4646 & 0,0472 \\
\hline EToL & EToT & $-0,1044$ & 2,6834 & 0,0826 \\
\hline
\end{tabular}

Observa-se na Tabela 2, o erro padrão de estimativa para cada estação do ano. Calculando-se o $\mathrm{S}_{\mathrm{y}-\mathrm{x}}$, de acordo com a expressão matemática apresentada na Eq. 9 deste trabalho, verificou-se que no verão, onde o referido erro foi menor, o mesmo foi de aproximadamente 7,7 \% constatando-se, desta forma, a aplicação do método de estimativa proposto (ETo), principalmente nesta época do ano.

Tabela 2. Erro padrão de estimativa $\left(S_{y-x}\right)$ para cada estação do ano

\begin{tabular}{cc}
\hline Estação & $\mathbf{S}_{\mathbf{y - x}}(\%)$ \\
Verão & 7,7 \\
Outono & 13,0 \\
Inverno & 15,7 \\
Primavera & 14,0 \\
\hline
\end{tabular}

\section{CONCLUSÕES}

1. As correlações entre as estimativas de evapotranspiração de referência, a EToT, em função da EToH, foi a que melhor se ajustou, principalmente no verão em que o coeficiente de determinação foi de aproximadamente 0,98.

2. O método de estimativa encontrado neste trabalho poderá subsidiar, após alguns ajustes, atividades agrometeorológicas, em especial aquelas inerentes ao crescimento, desenvolvimento e produção de culturas irrigadas.

3. Finalmente, recomenda-se a utilização do método empírico da estimativa de evapotranspiração de referência de Campina Grande, PB (EToT), para trabalhos de estimativas de evapotranspiração de referência não só para o município 
de Campina Grande mas, também, para regiões circunvizinhas ou de climas semelhantes.

\section{LITERATURA CITADA}

Allen, R. G.; Jensen, M. E.; Wright, J. L. Operational estimates of reference evapotranspiration. Agronomy Journal, Madison, v.81, n.4, 1989, p.650-662.

Allen, R. G.; Pereira, L. S.; Raes, D.; Smith, M. Crop evapotranspiration. Guidelines for computing crop water requirements. Rome: FAO, 1998, p.300. Irrigation and drainage paper 56.

Allen, R. G.; Smith, M.; Pereira, L. S.; Perrier, A. An update for the calculation of reference evapotranspiration. ICID Bulletin, New Delhi,v.43, n.2, p.35-90,1994.

Doorenbos, J.; Pruit, W. O. Guidelines for predicting crop water requirements. Rome: FAO, 1977, p.198. Irrigation and drainage paper 24

Hargreaves, G. H. Estimation of potential and crop evapotranspiration. Transactions of the ASAE, Saint Joseph, v.17, n.4, p.701-704, 1974.

Linacre, E. T. A simple formula for estimating evapotranspiration rates in various climates, using temperature data alone. Agricultural Meteorology, v.18, p.409-424, 1977.
Oliveira, A. D.; Volpe, C. A. Estimativa da evapotranspiração de referência utilizando dados de estação meteorológica convencional e automática In: Congresso Brasileiro de Agrometeorologia, 13, 2003, Santa Maria. Anais... Santa Maria: SBA, 2003. CD Rom.

Penman, H. L. Evaporation, transpiration and evapotranspiration. In: Vegetation and hydrology. Farnham Royal: Commonwealth Agricultural Bureaux., 1963, p.30-50.

SAS Institute. SAS/STAT user's guide. 5.ed. Cary: Statical Analysis System Institute, v.1, 1990, p.956.

Silva, V. P. R.; Dantas, R. T.; Campos, J. H. B. C.; Guedes, M. J. F. Estimativa da evapotranspiração de referência pelos métodos de Penman-Monteith - FAO/56, Hargreaves e Tanque Classe A em períodos diários mensais. In: Congresso Brasileiro de Agrometeorologia, 12 e Reunião Latino-Americana de Agrometeorologia, 3, 2001, v.2, n.2, 2001, Fortaleza. Anais... Fortaleza: SBA, 2001. p.467-468.

Silva, V. P. R.; Filho, A. F. B.; Silva, B. B.; Campos, J. H. Avaliação de métodos de estimativa da evapotranspiração de referência. In: Congresso Brasileiro de Agrometeorologia, 14, 2005, Campinas. Anais... Campinas: SBA, 2005. CD Rom.

Spiegel, M. R. Estatística: Resumo da teoria, 875 problemas resolvidos, 619 problemas propostos. 1.ed. São Paulo: McGrawHill do Brasil, 1977, p.404. 